Litinfinite Journal

ISSN: 2582-0400 [Online]

CODEN: LITIBR

Vol-1, Issue-2 (December, 2019)

Page No: 37-44

DOI: $10.5281 /$ zenodo.3959332

Interview

\title{
Interview of the famous female impersonator of Indian Theatre: Chapal Bhaduri
}

\section{Supriyo Chakraborty}

Publisher, Litinfinite Journal of Arts, Humanities and Social Sciences

Entrepreneur, Digital Marketing Strategist and Play director

Certified Google and Microsoft Analytic

Email Id: sreesup@gmail.com

\begin{abstract}
:
The given interview delves deep into the performative and psychological aspects of a female impersonator. Chapal Bhaduri was born a boy and he had an enriched theatrical background in his home. In this interview he talks about his initial performance, the struggles of his later life, and his role-playing as a female impersonator. The area of gender studies and gender and performativity are replete with numerous nuanced variations in terms of understanding the impact on the actor/actress on stage. Alterations and changing roles played by a man who impersonates a woman on stage, is therefore, liable to have an expansive outreach altogether. In this interview, Bhaduri also laments the current condition of the Indian yatra and the different types of media that have taken a toll on the traditional forms of theatre.
\end{abstract}

Keywords: Female Impersonator, Indian English drama, Indian theatre, Performance Studies, Gender Studies

Chapal Bhaduri: Chapal Bhaduri is a legendary character in the field of Indian theatre and plays. He has been doing the role of a female impersonator in regional Yatras and folk theatres of Bengal. He was born into a family of theatre personalities -his father Tara Kumar Bhaduri and mother Prava Debi both were from theatre background. His voice has been bearing a female tenor and he has been working as a female impersonator in many of the prominent Indian regional plays. There have been multiple documentaries on him, and a renowned tele-film Ushnotar Janye has also paid tribute to this doyen of Indian regional theatre.

\section{সাক্ষাৎকার: চপল ভাদুড়ি}

চপল ভাদুড়ি বাংলা থিয়েটারে এবং সম্ভবত ভারতীয় থিয়েটারে সর্বশেম জীবিত মহিলা চরিত্রাভিনেত্রী, যিনি একজন পুরুয হয়েও মহিলা চরিত্রে অভিনয় করেছ্েেন। তিনি বাংলা লোকনাট্যর একরূপ যাত্রায় মহিলা বেশ ধারণ করেছিলেন। ২০১০ সালে তিনি একটি বাংলা ছ্বি আরেকটি প্রেমের গল্পতে অভিনয় করেছিলেন।

তিনি কলকাতার কালী দত্ত লেনে জন্মগ্রহণ করেছিলেন। নাট্যাভিনেতা তারাকুমার ভাদুড়ির এবং প্রেক্ষাগৃহের অভিনেত্রী প্রভাদেবীর কনিষ্ঠ সন্তান চপল ভাদুড়ি। পরে ১৯৩৯ সালে তাঁর পরিবার কলকাতার গোয়াবাগান এলাকায় চলে আসেন। তিন ভাই ও দুই বোন ছিল। অভিনেত্রী এবং সঙ্গীতশিল্পী, কেতকী দত্ত ভাদুড়ি তার কনিষ্ঠ বোন। মায়ের সহায়তায় তার অভিনয় জীবন মাত্র সাত বছর বয়সে শ্রীরঙ্গম থিয়েটারে [বর্তমানে উত্তর কলকাতায় অবস্থিত বিশ্বরূপা নামে থ্যাত] শুরু হয়েছ্লিল যেথানে তিনি শরৎচন্দ্র চট্টোপাধ্যায়ের ‘বিন্দুর 


\section{Litinfinite Journal}

ISSN: 2582-0400 [Online]

CODEN: LITIBR

Vol-1, Issue-2 (December, 2019)

Page No: 37-44

DOI: $10.5281 /$ zenodo.3959332

Interview

ছেলে’-তে অপূর্বর চরিত্রে অভিনয় করেছ্দিলেন। পরে ১৯৫৫ সালে তিনি আলীবাবা নামক বাংলা থিয়েটারে অভিনয় করেছিলেন যেথানে তিনি মর্ডিনার চরিত্রে রূপদান করেছিলেন।

আলীবাবায় একটি মহিলা চরিত্রে অভিনয় করার পরে তিনি বহু থিয়েটারে মহিলা চরিত্রে অভিনয় করেজ্েে। রাজা দেবিদাস, চাঁদবিবি, সুলতানাতে তাঁর অভিনীত ‘রাভিয়া’ এবং ‘কৈকেয়ী’ চরিএদুটি তথনকার সময়ে প্রবল জনগ্রিয় হয়েছিল। একসময় তিনি চগলরানি নামে বাংলার যাত্রায় শীর্মস্থান দথল করেন। ১৯60-এর দশকে তিনি বাঙালি থিয়েটারের সর্বাধিক বেতনের 'অভিনেত্রী' ছিলেন। চপল ভাদুড়ি ১৯৫৮ সালে নাট্যকোম্পানীতে যোগদান করেছিলেন। যাটের দশকের শেশের দিকে মহিলারা যখন প্রেক্ষাগৃহে অভিনয় শুরু করেছিলেন তথন তিনি আধুনিক প্রবণতা মোকাবিলায় নিজেকে একটি ভুল প্রান্তে পেয়েছিলেন এবং তাকে পদত্যাগ করতে হয়েছ্ছিল। এই সময়ে তিনি কমলা প্রোডাকশনের সাথে কাজ করেছ্ছিলেন। 2006 সালে নির্মিত একটি নাটক (অর্ধ-আश্যীববনীমূলক) রমনিমোহনে তিনি পুরুয চরিত্রে অভিনয় করেন। চপল ভাদুড়ি সম্ভবত এমন একজন জীবিত নক্ষএ, यিনি যাত্রা এবং নাটকের মঞ্চে সমান ভাবে নিজের প্রতিগত্তি বিস্তার করতে পেরেছ্নেন একজন মহিলা চরিত্রাভিনেত্রী হয়ে, যদিও বাসুবে তিনি একজন পুরুষ। চপল ভাদুড়ির সাথে একান্ত আলাপচারিতায় কিচ্দু প্রশ্ন-উত্তর উঠে এসেছে এখানে, যেগুলো কিছু ইতিহাস বহন করে। চপল ভাদুড়ীর সাথে সাক্ষাৎকারে শ্রীতন্বী এবং সুগ্রিয় চঞবর্তী।

“আমি চেয়ার ছাড়তেও পারি, আবার দথলও করতে পারি’... চপল ভাদুড়ি শ্রীতন্বীকে নিজের চেয়ার এগিয়ে দিয়ে নিজের বসার জায়গা ছেড়ে দিলেন। এরপর নিজের সম্পর্কে প্রশ্ন করাতে, তিনি বলতে শুরু করলেন তার বাল্যকাল, কৈশোর, এবং কি ভাবে তিনি চপল ভাদুড়ি থেকে হয়ে উঠলেন চপলরানী।

শ্রীতন্বীঃ প্রথম অভিনয়ের অভিজ্ঞতা কিরকম ছিল? মনে আছে এখনো?

চপল ভাদুড়ীঃ ১৯৪৬ সালে বিশাল দাঙ্গা হয়েছিলো, দেশ ভাগ হয়ে গেলো। পূর্ববঙ্গ হয়ে গেলো পূর্ব-পাকিস্তান, আর এটা গশ্চিমবঙ্গ। ১৯৪৬ সালে, ১৬ই জুলাই, বুধ্ববার, তারিখ আর দিনটা আমার এথনো মনে আছে। এই দাঙ্গার সময় বহু বনেদী পরিবার কলকাতা ছেড়ে চলে গেলো মধুপুর, গিরিডি এবং অন্যান্য জায়গায়। আমার নাটক দেখতে থুব ভালো লাগতো। বুঝতাম কতটুকু তা জানি না, কিন্ত দেখতাম, আর ভাবতাম আমিও যদি এরকম করতে গারতাম! কত গহনা, কত সাজগোশাক, তথন ঐতিহাসিক নাটকগুলো বেশি হতো। শরৎচন্দ্রের অনেক নাটক তথন হয়েছে। ‘বিন্দুর ছেলে” নাটকটিতে তথন তিনটে চরিত্র হতো, শিশু, বড় বয়স এবং বুড়ো বয়স। আমার বোন কেতকী দত্ত তথন প্রথম অভিনয় করে এই বড় বয়সের ছেলেটির চরিত্রে। গরে ওর চেহারা তাড়াতাড়ি বিকশিত হওয়ারফলে ওকে গিরিশচন্দ্রের ‘সিরাজউদৌল্লা' নাটকে লুৎফন্নেসা করতে হয়। সিরাজের চরিত্রে অভিনয় করেছিলো আমার ছোট কাকা মুরারিমোহন ভাদুড়ি|

তথন এতবার ‘বিন্দুর ছেলে দেথেছ্ যে পুরো নাটকটা আমার মুখস্থ হয়ে গিয়েছিলো। তথন রবিবার দুটো শো হতো, তিনটের সময় আর সাড়ে-ছটায়। শনিবার একটা আর বৃহস্পতিবার একটা করে শো হতো। একদিন দুপুরবেলা নাটকের একজন এসে বললেন “চলো, তোমাকে তোমার মা ডাকছ্নেন। “ “আমি জিজ্ঞেস করলাম মা ডাকছে কেন? আমি তো বাড়িতেই আছ্ছি, নাটক দেথতে যাইনি। “ নাটক দেথা মা পছ্দন্দ করতেন না। তথন সাবিত্রীদেবী নাটকে অভিনয় করতেন। আমাকে দেথে মাকে বললেন এই দেথো টুকু এসে গেছে। আমার ডাকনাম টুকু। মা বললেন- “কিরে, রোজ তো নাটক দেথিস, আমার কথা শুনিস না, বিশেষ করে এই নাটকটা বারবার দেখিস। জানিস, ছোট অমূল্যর পার্টটা যে করে, সেই ছেলেটি আজ আসেনি। তুই করতে 


\section{Litinfinite Journal}

ISSN: 2582-0400 [Online]

CODEN: LITIBR

Vol-1, Issue-2 (December, 2019)

Page No: 37-44

DOI: $10.5281 /$ zenodo.3959332

Interview

গারবি?” আমি বললাম-“ছ্যাঁ, গারবো’। তথন আর ১৫ মিনিট বাকি শো শুরু হতে। বিন্দু মানে, সাবিত্রীদেবী স্টেজে উঠলেন, তার প্রথম সংলাগ-“দিদি, ও দিদি, আজকে বুঝি অমূল্য প্রথম পাঠশালায় যাচ্ছে?” অন্নপূর্ণা বললো-‘‘্যাঁ, ঠাকুরঝি ওকে আজ থেকে পাঠশালায় পাঠাচ্ছি|আশীর্বাদ করো, যাতে ও মানুয হয়। “

এবার আমার সংলাগ। কিন্ত সব ভুলে গেলাম, সামনে দর্শক দেথে মনে হছে যেন পুরী'র অন্ধকার সমুদ্র। কি রকম একটা লাগছ্,ে, মনে হছে বিরাট ঢেউ, চারিদিক অন্ধকার। আমি বলে উঠলুম-“তুমিও চলো না ছোটমা’। তথন একজন বড় অভিনেতা ছোট অভিনেতাকে চালিয়ে নিয়ে যেতে গারত, এখন কোন অভিনেতার সেই ক্ষমতা নেই। এরগর, স্মারক যা বলতে থাকলো, আমিও সেই মতো বলতে লাগলুম। এই আমার প্রথম অভিনয়। এরগর, মাকে বললাম নাটক দেথতে যাবো, কিন্ত মা বারন করলেন। বললেন-“যা পেয়েছো, অনেক পেয়েছো।" বললাম- “কি পেলাম মা?” বললেন “তুমি বড় হও, তথন বুঝতে গারবে কি পেলে বা কি না পেলে। “আমার ভালো লাগছিলো না। তথন আমার ন-কাকা আমাকে ডাকলেন। আমার বাবা-কাকারা ছিলেন নয় ভাই, সবাই এই কাজের মধ্যে যুক্ত। ন-কাকা বললেন-"টুকু এদিকে যায়, এই ভাউচারটায় সই কর। আমি তথন অনেক ছোট, সই করতে হয় কি করে বুঝি না। বললেন আমার নামটা লিথতে। লিথলাম -"চপল ভাদুড়ী"।

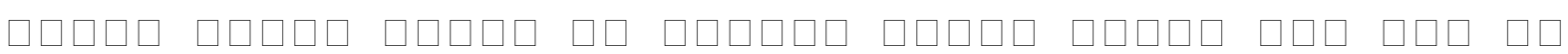

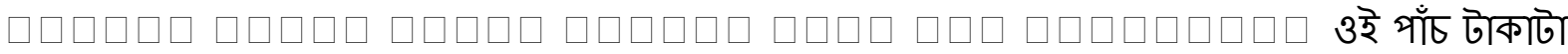
আমার জীবনের প্রথম উপার্ডন।

মা ফিরে এসে বললেন, আমার অভিনয় ভালো হয়েছে। জিজ্ঞেস করলাম- "তাহলে বৃহস্পতিবার যাবো তো?" বললেন- "সে পরে দেখা যাবে"।

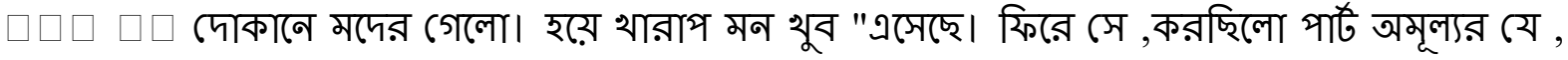
এ যদি কাউকেকবার মদ থাইয়ে দাও, তাইলে সে মদ ছাড়া থাকতে পারে না, বারবার সেই নেশাটা করতে চায়। অভিনয়ের নেশাটাও অনেকটা সেইরকম। দর্শকদের থুশি করার নেশা, চমক দেওয়ার নেশা। পরেরদিন আমি আবার অভিনয় দেখতে চলে গেলাম।তথনতো আমাদেরই সব, আমি বক্সে বসে নাটক দেখতে লাগলাম। মা রাগ করলেন, বললেন - "তুমি এলে কেন, বারণ করেছ্ছিলাম না?" বললাম- "মনটা ভালো লাগছ্ছিলো না।" মা বললেন - "ঠিক আছে, এইখানে নিচে বসে দেথো|" মা আমাকে চোথের আড়াল করতেন না। তথন আমার বয়স সাত।

তথন মা অনেক নাটক করেছ্নেন। রবীন্দ্রনাথের শেষরক্ষা, এবং বহু পৌরাণিক নাটক। আমি সেগুলোকে গ্রামাফোনে চালিয়ে চালিয়ে শুনতাম। আমার তো সেইরকম কোনো বন্ধু-বান্ধব ছিল না। ক্রিকেট, ফুটবল থেলা দেখতুম, আমার প্রিয় থেলা ছিল ফুটবল। কিন্ত কোনোদিন থেলিনি। ফুটবল পচ্দের থেলা হলেও, ক্রিকেটের অধিপতি সৌরভ গাঙ্গুলীকে আমি মনে মনে থুব পছ্ন্দ করি। দুঃথের বিষয়, তাকে কোনোদিন আমি চাক্ষুষ করতে পারলুম না। ওনার প্রতি আমি থুবই আকৃষ্ট।

শ্রীতন্বীঃ অমূল্যর গরে আপনার দ্বিতীয় নাটক কবে মঞ্চস্থ হলো?

চপল ভাদুড়ি: ১৯৪৭ সালে, ১৫ই অগাস্ট দেশ স্বাধীন হলো। সেদিন দেশের প্রতিটি কোনা, এমনকি থিয়েটার বোর্ডগুলোও সাজানো হয়েছ্ছিল। ফুলের ব্যবহার তথন তেমন ছিলো না, আমরা কাগজের শিকলি বানিয়ে সাজাতাম। আমি পড়তাম টাউন স্কুলে, এই শ্যামনগর স্ট্রিটে। সেদিন দেশে উৎসবের মেজাজ, চারিদিকে গান 


\section{Litinfinite Journal}

ISSN: 2582-0400 [Online]

CODEN: LITIBR

Vol-1, Issue-2 (December, 2019)

Page No: 37-44

DOI: $10.5281 /$ zenodo.3959332

Interview

চলছ্,ে, হিন্দী গান নয়। তথন বিথ্যাত গায়ক সত্য চৌধুরীর গান "পৃথিবী আমাকে চায়" থুব জনগ্রিয়তা লাভ করেছ্ছিল। গিরি চঐবর্তী অনেক গুলো কাজী নডরুল ইসলামের গান গের্েেছিলেন। স্বাধীন দেশে অনেক থিয়েটার থুলতে লাগলো, কিন্ত আমার আর কিছ্ডু হলো না। আমি বাড়িতে বসে পড়াশুনো করছি। বাংলা ভাযায় দক্ষতা ছিলো, ইতিহাস এবং বিজ্ঞানেও ভালই ভালো ছিলাম। কিন্ত অঙ্কে একেবারে মাটি। এই সময় মা দল ছাড়লো। মা ৫০ টাকা পেতো মাসে। শিশিরকুমার ভাদুড়ীকে বলেছিলেন আরো ৫০ টাকা বাড়াতে। কিন্ত তথন ওনার আর্থিক অবস্থা পরিবর্তন হচ্ছে। উনি মাকে বললেন, উনি আর টাকা বাড়াতে পারবেন না। কিন্ত মা অন্য কোনো দলে যোগ দিতে পারে, রেবা মায়ের জায়গাটা চালিয়ে দিতে পারবে।

রেবাদেবী মায়ের সমকক্ষ না হলেও, অনেকটা মায়ের মতো অভিনয় করতেন। তথন ছবি বিশ্বাস মিনার্ভা থিয়েটার লিজ নিলেন পাঁচ বছরের জন্য। যদিও উনি এক বছরের বেশি চালাতে গারেননি। সেই সময়, ছবি বিশ্বাসের "উদয় সিংহ" নাটকে আমি করলাম উদয় আর মাধু, অর্থাৎ মাধবী করলো কনক। সেই থেকে মাধবীর সাথে আমার থুব বন্ধুত্ব, যা এখনো অটুট। মাধুকে এখনো দেখা হলে জিজ্ঞেস করি, মনে আছ্ মাধু, তমলুকে অভিনয় করতে গিয়ে কিরকম আমার গলায় ফাঁস লেগে গিয়েছ্ছিলো?

শ্রীতন্বীঃ এই নাটকটির প্রথম অভিনয় কি তমলুকে হয়?

চপল ভাদুড়ি: না, প্রথম মঞ্চস্থ হয় মিনার্ভাতে, পরে তমলুক এবং অন্যান্য জায়গায় শো হয়। এই ধাত্রীপান্নায় অভিনয় করতেন তথনকার দিনের বিরাট অভিনেত্রী সরযূবালা দেবী। বনবিবীর পার্ট করতেন ছবি বিশ্বাস, আমার বোন চম্পা বলে একটা ছোট চরিএ করতো। আর আমার মা করতেন বনবিবীর মায়ের চরিত্র, থুব ডার্ক গার্ট|

একদিন সরযূমাকে বলতে শুনেছি (তথনকার দিনে, সিনিয়র অভিনেত্রীদের আমরা মা বলে ডাকতাম), তিনি আমার মাকে বলজ্নেন - "দিদি আমি কিন্ত তোমার চোথ দুটো গেলে দেবো, নয়তো থেয়ে নেবো। কারণ তুমি এমনভাবে সংলাপ বলো, আমি দেখতে পাই যে, তোমার দু-চোথে আগুন ড্বলছে। "

এরপর ছবি বিশ্বাসের দল এক বছ্রর পরে বন্ধ হয়ে যাওয়াতে, মা "রঙমহলে" এলেন। ১৯৫০ সালে মা রঙমহলে এলেন, আর ওই সালেই মা সরোজবাবুর "নিষ্কৃতি" করলেন। আমার ভাগ্য থুলে গেলো। এই নাটকে আমি শৈলজার, অর্থাৎ সতীনের ছেলের পার্ট করলুম। এই নাটকে সুখেন দাস অতুলের চরিত্র করতেন। ভালো চলেছিলো নাটকটা, পুজোর সময় টানা চারদিন শো হতো। এরপরে, ১৯৫২ সালে "সেই তিমিরে" বলে একটি নাটক করলাম। সাতটা বৌ, তাদের স্বামীদের ঔদ্ধত্ব সহ্য করতে না পেরে, সংসার ছেড়ে একটা মেস-বাড়িতে থাকবে। সেই মেসবাড়ির ঝিয়ের পার্ট করতো আমার মা, কমেডি চরিত্র। ১৯৫২ সালের ৬ই নভেম্বর এই নাটকটির পুনঃপ্রকাশ হলো। মা তথন গণনাট্য সংঘে যোগ দেওয়ার জন্যে প্রস্তুত। কিন্ত কোনো এক কারণে মা সেদিন অভিনয় করতে পারলো না। ৮ই নভেম্বর সকালে মায়ের চিৎকারে ঘুম ভেঙে গেলো। মা সকালে কাজের লোককে চা বসাতে বলে গান মুথে দিয়ে বসেছে। আমাদের পরিবারে পানের থুব চল ছিলো, আর ছিল পানীয়। ভাদুড়ি পরিবারকে লোকে পানীয় বংশ বলে চিনতো। মা বাবাকে বললেন - "আমার শরীর থারাগ লাগছে, ছোটুকে ডাকো, ছোটু আমার ছোটদি। মা থুব ছটফট করতে লাগলো, আমি বুঝতে পারলাম না, ভাবলাম মা কি অভিনয় করছে? ওই প্রথম মৃত্যু দেখা আমার চোথে, নিজের মায়ের মৃত্যু। সবাই কান্নাকাটি করছে, বলছে মা নেই। কিন্ত আমার কোনো কান্নার বেগ এলো না। আমার মা মরে যাবে? সে কি করে হয়? মাকে শেষ শ্রদ্ধা জানাতে অনেকে এসেছিলেন, কাননদেবী তাদের মধ্যে একজন| আমি তথন কাননদেবীকে 


\section{Litinfinite Journal}

ISSN: 2582-0400 [Online]

CODEN: LITIBR

Vol-1, Issue-2 (December, 2019)

Page No: 37-44

DOI: $10.5281 /$ zenodo.3959332

Interview

দেখতে ব্যস্ত। মাকে সাজিয়ে নিয়ে যাওয়া হলো। সবাই জোর করে আমাকে দিয়ে মুখাগ্নি করালো, মায়ের মুখটা আমার সামনে পুড়ে যাবে এটা দেখতে দেখতে আমি কান্নায় ভেঙে গড়লাম। শুধু বলতে লাগলুম, মা চলে গেলো, অন্নপূর্ণা চলে গেলো।

পড়াশুনা বেশিদূর হলো না, থেলাধুলো করতাম না, বন্ধু-বান্ধব বিশেষ ছিলো না। গান গাইতাম, লতা মঙ্গেশকর আমার গ্রিয় শিল্পী। সারাদিন কাটতো বাড়ির কাজকর্ম করে, বাজার করা, ছাই-থোল দিয়ে বাসন মাজা, জল তোলা। মায়ের কাছে তো অভিনয় শিখতে গারিনি, তাই মায়ের অভিনয়ের ছবিগুলো দেথে পরবর্তীকালে শেখার চেষ্টা করেছি।

তথন আমার পনেরো বছ্রর বয়স। সংসারটা ভেঙে গেলো, আমার বড়-ডামাইবাবু আমাকে বললেন - "কিরে কিছ্ করতে হবে তো? না হলে চলবে কি করে?" বললাম- "আমি তো লেথাপড়া কিচ্ডু করিনি, কি করবো জামাইবাবু?" উনি বললেন, তোর দ্বারা কোনো কাজ হবে না। বললেন - "মর্জিনার পার্ট করবি?" বললাম"সে কি করে সম্ভব? আমি তো পুরুষ, আমি কি করে নারীচরিত্র করবো?" উনি বললেন - "ঠিক পারবি। তোর কন্ঠ ভালো, তোর গলা, নৃত্য-এসব কাজে লাগাবি। অভিনয় করবি। বাকি ডিরেক্টর শিখিয়ে দেবে। यদি করিস তাহলে তোর ইস্টার্ন রেলওয়েতে একটা চাকরি হবে। " ওনার কথা মতো জ্যোতিদার কাছে গেলাম, উনি ডিরেক্টর ছিলেন। উনি আমাকে দেথে আমার জামাইবাবুকে বললেন - "এই কাকে এনেছ্ছিস পঙ্কজ, রোগা, পাতলা, কালো, এ কি করতে পারবে? উনি আমাকে একটা গান গিয়ে বললেন, "ভালোবাসি তাই ভালোবাসিতে আসি"।

$\square \square \square \square \square$

पिएव

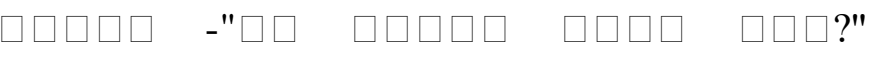

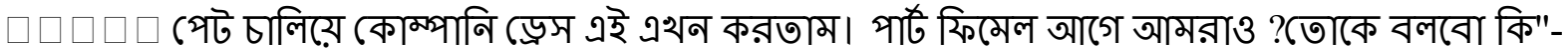

"চালাই। আমাকে মেকআপ করে দিলো, পোশাক পরিয়ে দিলো, লাল টকটকে ইরানি পায়জামা। ন্যাকড়া দিয়ে দুটো বল বানিয়ে দড়ি দিয়ে বেঁধে আমার ব্রেস্ট তৈরী করা হলো। সোনার গহনা পরালো। বাঁদীরা সোনার গয়না আর বেগমেরা ইীরের গয়না গরতো| নিজেকে আয়নায় দেথে চিনতে গারলাম না। দেখলাম আমার গিচ্ননে অনেক কম বয়সী ছেলেরা দাঁড়িয়ে আমাকে দেথছে। ওদের মধ্যে একজন এগিয়ে এসে আমাকে বললো "থুব সুন্দর দেখাচ্ছে তোমাকে। কিন্ত গোলাশ ফুলটা লাগালেনা না কেন?" কোথা থেকে একটা গোলাগ এনে আমার থোঁগায় পরিয়ে দিলো সেই ছেলেটি।

এই মর্ডিনা করে ৫০ টাকা পেলাম। এরগর রেলে কাজ পেলাম চেনম্যান হিসেবে। একদিন অফিসের একজন এসে বললেন আমাকে নাকি টাইপ সেকশনে ডাকছে। গিয়ে দেথি সেই ছেলেটা, যে লাল গোলাপ পরিয়ে দিয়েছ্ছিল আমাকে। বললো-"তোমাকে ঐসব কাজ করতে হবে না। রোজ দুগুরবেলা সময় করে টাইপ সেকশনে চলে আসবে, টাইপ শিখবে। পরে আমরা তোমাকে টাইপ সেকশনে নিয়ে নেবো। কিন্ত তাও আমার দ্বারা হলো ना।

সুপ্রিয়: আপনি রুপান্তরকামী, নারী, না পুরুষ? নিজেকে কি বলে পরিচয় দিতে বেশি স্বচ্ছন্দ বোধ করেন?

চপল: আমি পুরুষ। কিন্ত মেকআপ করার পরে, যথন আমি চুল আর মেয়েদের (োশাক ধারন করি, তথন আর নিজেকে পুরুষ বলে ভাবতে পারি না। এমনকি তথন কোনো পুরুযের দিকে আমি তাকাইওনা, কারণ আমি পুরুষদের চোথের ভাষা পড়তে পারি তথন| 


\section{Litinfinite Journal}

ISSN: 2582-0400 [Online]

CODEN: LITIBR

Vol-1, Issue-2 (December, 2019)

Page No: 37-44

DOI: $10.5281 /$ zenodo.3959332

Interview

সুপ্রিয়: উত্তমকুমারের সাথে আপনার পরিচয় কি ভাবে?

চপল: ১৯৬৮ সালের ঘটনা। রবীদ্রসদনে মাইকেল মধ্সুদূদ নাটক হচ্ছে। আমি জাহৃবীর পার্ট করছি। তথন আমার বয়স ৩০-৩২ হবে। নাটকটার ডিরেক্টর ছিলেন স্বগনবাবু। জাহ্বী আমার ডীবনের অন্যতম শ্রেষ্ঠ চরিত। লালপাড় শাড়ি, সোনার গহনা, নাকছ্দাবি, চুনী-পান্না দিয়ে সাডানো হয়েছ্ছিল আমাকে। নাটকটিতে থুব ছোট পার্ট ছ্নিল আমার। মাইকেল যথন খ্রিস্টান হয়ে যাচ্ছে, শেষ দৃশ্যে জাহুবীর পুত্র হারানোর বেদনায় দুচোথ দিয়ে জল পড়ছে, আর কঠিন কষ্টের জন্ম নিচ্ছে আমার দুচোথে। গরে জাহ্বী পাগল হয়ে গেলো, এবং কারো মা ডাক শুনলেই ভাবতে লাগলো তার পুত্র মধু তাকে ডাকছে। শেষ সংলাপে রূপনারায়ণ দত্তকে জাহৃবী বলছে -"কে গান শোনাবে? নয়ন? নয়ন তো অন্ধ হয়ে গেছে। " এই বলে জাহুবী নিজের আঁচল কে মধু তেবে বলতে লাগলো - "আয় চাঁদ, আয় চাঁদ টিপ দিয়ে যা।।।" এই বলে জাহৃবী চিৎকার করে স্টেজে গড়ে গেলো, মৃত্যু হলো জাহুবীর।

এই দৃশ্য দেথে উত্তমবাবু আমাকে ডেকে পাঠালেন। উনি জানতেন না, জাহ্বী একডন পুরুষ। আমাকে দেথে এক ভদ্রলোককে বললেন - "এ না, জাহৃবীকে ডাকো"। $\quad \square \square \square \square \square \square \square$ - " $\square \square \square \square$ হৃবী, চপলরানী, কেতকী দত্তের ভাই"।

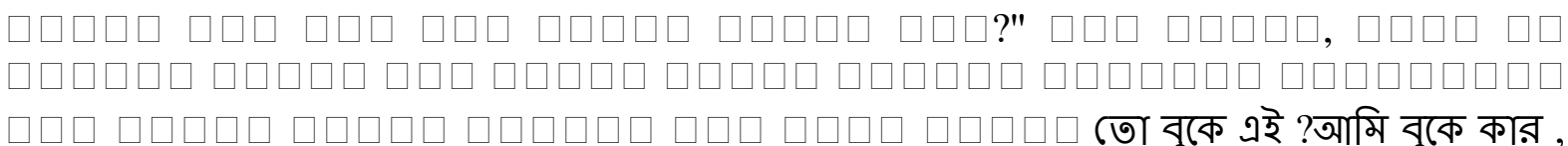
সাবিত্রীর, সুপ্রিয়ার, সুচিত্রার থাকার কথা। উনি বললেন - "কি চাও তুমি? বলো কি চাও আমার থেকে?" আমি বললাম, কিষ্ছু না, শুধু আশীর্বাদ করুন যাতে এরকম ভাবে অভিনয় করে যেতে পারি।

সুপ্রিয়: নাটক থেকে শুরু করে যাত্রায় এলেন কেন?

চপল: দুবচ্মর রেলে কাজ করেছিলাম, কিন্ত সেখানে শুধু অভিনয় করতে হয়েছে, কাজ বিশেশ কিছু করতে হয়নি। তারপর সত্যব্রত রায় আমাকে একদিন বললেন শৌখিন অভিনয় করবি? দেওঘরে অভিনয় করতে যাচ্ছি, গুজোর সময়। 'সম্রাট অশোক' মঞ্চস্থ হলো। তথন বনফুল নামের একটি ছেলে এসে আমাকে বললো "বিশাখা যাত্রাদলে কাজ করবে?" সে প্রথম আমাকে যাত্রাদলে কাজ করবার প্রস্তাব দিলো। বনকুলের আসল নাম কানাই দাস|যাত্রাদলে সবার নাম পরিবর্তন করা হতো। ওদের দল থেকে ছবিরানী চলে গিয়েছিলেন। আমি ছবিরানীর জায়গা নিলাম। ১০০ টাকা মাইনে। কিন্ত আমার নাম কোনো পালায় পড়তো না। পরে সূর্যবাবুকে বললাম শিলিগডড়িতে - "সবাই বলছে আমি ভালো অভিনয় করছ্,ি, তাতেও আমার নাম পড়ছে না কেন?" গরে ‘রমণীমোহন’ করে আমি প্রথম স্বীকৃতি পেলাম। ১৯৬০ সালে আমাকে নিতে ব্রজেন্দ্রকুমার দে আমাকে নিয়ে "চাঁদবিবি" করলো। এই চাঁদবিবি করে চপলরানীর নাম সবাই জানতে পেলো। এরপর রাভিয়া সুলতানা করলাম ১৯৬৬ সালে।

সুপ্রিয়: সিনেমা করতে ইচ্ছে হয়নি কখনো?

চপল: একজন পুরুষকে সিনেমায় নারীচরিত্রে নেবে কেন? সিনেমায় সেরকম সুযোগ কোথায়? রাডিয়া সুলতানা করার পরে নাট্যকোম্পানির সাথে আমার মতবিরোধ হয়, পরে আমি অন্য দলের হতে মধুসূদন 


\section{Litinfinite Journal}

ISSN: 2582-0400 [Online]

CODEN: LITIBR

Vol-1, Issue-2 (December, 2019)

Page No: 37-44

DOI: $10.5281 /$ zenodo.3959332

Interview

করি। ‘উপল ভাদুড়ী’ নামে একটি নাটক আমার জীবন অবলম্বনে করা হয়েছে। আমার যৌবনের গার্টটা করেছে রঞ্জন ঘোম। আমার সাথে সমান তালে অভিনয় করার মতো ক্ষমতা একমাত্র রঞন ঘোমের আছে। এমনকি কোনো মেয়েও চপলরানীর মতো অভিনয় করতে পারবে না। পরে যাতায় মেয়েরা আসতে শুরু করলো, আর আমার কদর কমতে শুরু করলো। ছয় হাভার টাকা মাস মাইনে পেতাম, সেটা পরে কমতে কমতে ৩৫০ টাকা হলো।

সুপ্রিয়: যাটের দশকে চপলরানী মঞ্চ কাঁপাচ্ছে, কিন্ত আপনার কোনোদিনও মনে হয়েছে সমাজ আপনাকে পুরুষ নয়, নারীরূপে বেশি গ্রহন করছে?

চপল: আমি কোনোদিন কোনো বাধার সম্মুথীন হইনি। আমি যেহেতু কেতকী দত্তের ভাই, মায়ের ছেলে, কমল দত্তের ভাই, তাই গাড়ায় কোনো অসুবিধে হতো না। সবাই মাঠে থেলতো, আমি দেথতাম।

সুপ্রিয়: থেলাধুলো করলেন না কেন? ছোটবেলা থেকে feminine ছিলেন বলে?

চপল: মনে হয়। আমি ঘরটাকে বেশি ভালোবাসতাম। পুতুলথেলা, রান্নাবাটি বেশি ভালোবাসতাম। ফুটবল বা ক্রিকেট নয়।

সুপ্রিয়: স্টেজে চপলরানীকে দেথে বিবাহিত পুরুষদের কি তাদের স্রীয়েরা আড়াল করার চেষ্টা করতেন?

চপল: টিটুদা ছিলো আমার দাদার বন্ধু। যার্যায় ব্যস্ত থাকার কারণে ওর বিয়েতে যেতে পারিনি। একদিন ছোটদি বললো টিটুদা আমাকে নাকি থুঁজছে। সন্ধ্যাবেলা টিটুদা বাড়িতে এসে বললো - "কিরে, তুই আমার সংসারটা এরকম ভাবে ভেঙে দিবি?" আমি তো অবাক। জিজ্ঞেস করলাম কি ব্যাগার। বললো, টিটুদা আমার সাথে তোলা একটা ছবি আলমারিতে রেথে দিয়েছিলো। মনে মনে (েবেছিলো, কোনোদিন বিয়ে করলে ওর বৌ এরকম দেখতে হবে। সেই ছবি দেথে ওর নতুন বৌ ওকে এখন ছেড়ে দিতে চাইছে। আমি বললাম, তোমার বাড়ি যেতে গারবো না। তুমি বৌদিকে নিয়ে আমার যাত্রা চাঁদবিবি দেথতে এসে, আর গালা শেম হলে গ্রীনরুমে আমার সাথে দেথা করে যেও। পালা শেযে বৌকে নিয়ে টিটুদা গ্রীনরুমে এলো। আমি তথনও আমার পোশাক ছ্যাড়িনি। বৌদিকে জিজ্ঞেস করলাম কি ব্যাপার? কেমন আছ্নেন? উনি বললেন- "কেন এরকম করলেন আমার সাথে? আমার জীবনটা শেম করে দিলেন?" আমি তথন মাথার চুলটা থুললাম। উনি দেথে অবাক। বললাম, যান এবার আমার দাদাকে নিয়ে রাত্রিটা শান্তিতে কাটান।

সুপ্রিয়: শিশির কুমার ভাদুড়ির বংশধর হয়ে, মহিলা চরিত্রে অভিনয় করলেন। নাট্য-জগৎ থেকে বঞ্চনার শিকার হতে হয়নি?

চপল: সেরকম অসুবিধের মুথে পড়িনি কথনো। কিন্ত আমার পরিবারের একজন আমাকে এক সময় অনেক অপমান করেছ্নেন। উনি আজ নেই। কিন্ত আমি তো লন্ডন-কানাডা সব দেশে অভিনয় করে দর্শকদের মন জয় করেছে। আমি যা পেয়েছ্ছি, সেটা অন্য কেউ পায়নি।

সুপ্রিয়: চপলরানী তৈরী করার পেছ্নেে কার অবদান সবথেকে বেশি? 
Litinfinite Journal

ISSN: 2582-0400 [Online]

CODEN: LITIBR

Vol-1, Issue-2 (December, 2019)

Page No: $37-44$

DOI: $10.5281 /$ zenodo.3959332

Interview

চপল: তিনজন, মাথনলাল থট্টর, সূর্য কুমার দত্ত আর ব্রজেন্দ্রকুসার দে। ওনারা না থাকলে আজ চপলরানী থাকতো না। "থোলা জানালা, বন্ধ চোথ" বলে একটি নাটক করেছিলাম, ভর্গনাথ ভট্টাচার্য ডিরেক্টর ছিলেন। ৩০ বছর ধরে একজন পুরুষ নারী চরিত্রে অভিনয় করে, শেষ দৃশ্যে সে বলছে- "আমি সেই অ্বলন্ত ব্যাধির শিকার, যার জন্ম আমি দিইনি|"

এই বলে নিডের সব আবরণ এক এক করে থুলে ফেলছে সে, আর ধীরে ধীরে পুরুষ রূপ ধারণ করছে...

Note: This is an interview conducted in Bengali and there is no citation or works cited required. It is a live interview taken by Supriyo Chakraborty, Publisher of Litinfinite Journal. 